\title{
¿Es Despistado o Está Despistado?: Infiriendo la Estabilidad de los Rasgos Psicológicos
}

\section{Is He Spaced Out (Just Now) or Is He (Always) Spaced Out? Inferring the Stability of Psychological Traits}

\author{
Paula Alonqueo \\ Universidad de La Frontera
}

\author{
Pilar Soto \\ Universidad Autónoma de Madrid
}

\begin{abstract}
Un rasgo psicológico descrito con el verbo ser se considera más estable que uno descrito con estar (Heyman \& Diesendruck, 2002). Para establecer si esta distinción lingüística afecta a las inferencias sobre características personales se realizaron 2 estudios complementarios con 62 escolares en diferente nivel de desarrollo (7 y 12 años) y 108 estudiantes universitarios monolingües de español peninsular, de nivel socioeconómico medio, residentes en Madrid, España. En ambos los participantes se distribuyeron en dos condiciones experimentales (ser y estar) y respondieron a una tarea de inferencias en que se describían características psicológicas y conductas asociadas a estas. Se realizaron análisis multivariados y de medidas repetidas considerando la edad y la condición experimental. Solo los adultos y los niños mayores infirieron que los rasgos descritos con ser eran más estables que los descritos con estar, pero los menores en ambas condiciones experimentales infirieron que los rasgos eran estables. El efecto de ser y estar en las inferencias es relevante, pero debe interpretarse considerando las diferencias evolutivas.
\end{abstract}

Palabras clave: inferencias, lenguaje y cognición, verbos copulativos

\begin{abstract}
A psychological trait described with the verb ser is considered more stable than one expressed by using estar (Heyman \& Diesendruck, 2002). In order to establish whether serlestar verbal forms affect inferences about psychological traits, 2 studies were carried out on school children in different developmental levels ( $N=62,7$ and 12 years) and university students $(N=108)$, monolingual speakers of Peninsular Spanish, from medium socioeconomic status, resident in Madrid, Spain. In both studies participants were randomly assigned to one of 2 experimental conditions (ser and estar) and responded to an inference task which consisted of the description of psychological characteristics and behaviors associated with them. Factorial and repeated measures analysis of variance were performed. Only the adults and older children inferred that the traits described with ser were more stable than those expressed with estar. The younger children inferred that the traits were stable in both experimental conditions. The effect of the linguistic distinction on inferences is significant, but should be interpreted bearing developmental differences in mind.
\end{abstract}

Keywords: inferences, language and cognition, copulative verbs

Las investigaciones translingüísticas realizadas en las últimas décadas sobre distintos sistemas semánticos han puesto de manifiesto la existencia de importantes diferencias en la forma en que las lenguas analizan y organizan el mundo (véanse en Dasen \& Mishra, 2010 y en Guo et al. 2009). Los datos empíricos obtenidos en dichos estudios han dado lugar al resurgimiento del antiguo debate - la hipótesis del relativismo lingüístico planteada por Sapir (1949) y Whorf (1956) — sobre las relaciones entre lenguaje y pensamiento.

Paula Alonqueo Boudon, Departamento de Psicología, Universidad de La Frontera, Temuco, Chile; Pilar Soto Rodríguez, Departamento de Psicología Evolutiva y de la Educación, Universidad Autónoma de Madrid, España.

En este artículo se presenta parte de los datos de la tesis doctoral de la primera autora, dirigida por la segunda. Esta investigación fue posible gracias a la beca para estudiantes extranjeros de la Agencia Española de Cooperación Internacional y la Beca Presidente de la República del Ministerio de Planificación y Cooperación de Chile, ambas otorgadas a la primera autora.

Agradecemos especialmente los comentarios de Miguel Galeote, Virginia Gathercole, Amparo Moreno, Miguel Pérez-Pereira, Eugenia Sebastián, así como a Pedro Guijarro-Fuentes y Carmen Silva-Corvalán.

La correspondencia relativa a este artículo debe ser dirigida a Paula Alonqueo Boudon, Departamento de Psicología, Universidad de La Frontera, Francisco Salazar 01145, Temuco, Chile. E-mail: palonque@ufro.cl 
Desde una perspectiva actual, no se puede establecer una relación directa y universal entre las categorías conceptuales y su expresión en el lenguaje, pues algunos conceptos reciben una expresión lingüística y otros no. Los primeros son distinciones relevantes para los hablantes de una lengua y constituyen una fuente de significado compartido (Gelman \& Kalish, 2006), expresado mediante construcciones gramaticales que utilizan diferentes elementos (e.g., morfemas de género, número, tiempo, aspecto). Algunas de estas nociones gramaticalizadas parecen ser universales, mientras que otras se observan solo en algunas lenguas (Talmy, 2000).

Existe una gran variabilidad en el tipo de nociones que gramaticalizan las diferentes lenguas (Slobin, 2009). Por ejemplo, Lucy (1992) y Lucy y Gaskins (2003) estudiaron las diferencias lingüísticas entre el inglés y el maya yucateco y su influencia en la resolución de tareas cognitivas. Ambas lenguas difieren en la marcación del plural, obligatoria en la primera, opcional en la segunda. Además, en inglés las entidades contables son modificadas directamente por los numerales (e.g., una taza, dos tazas), pero en maya yucateco los modificadores numerales se acompañan de un clasificador numeral que informa sobre la composición material de los objetos (e.g., una vela de cera). Estas diferencias lingüísticas influyeron en la resolución de una tarea de categorización de objetos que los hablantes de maya yucateco clasificaron según su composición material y los hablantes de inglés, según su forma.

La proyección precisa entre categorías conceptuales y gramaticales supone un aprendizaje, pues los niños deben descubrir las nociones codificadas en su lengua materna y cómo se gramaticalizan (Bowerman, 1996). Por ello, el debate actual sobre las relaciones entre lenguaje y pensamiento se centra en el aprendizaje y desarrollo del lenguaje (véanse en Bowerman \& Levinson, 2001 y en Gentner \& Goldin-Meadow, 2003).

Es posible que el desarrollo cognitivo condicione la aparición de formas lingüísticas concretas. Según Clark (2001), se comienza con una serie de categorías conceptuales muy generales y sobresalientes que funcionan como hipótesis para asignar significados. Si dichas categorías reciben una expresión convencional en una lengua (e.g., la marcación de singular-plural), se aprenderán rápidamente; son categorías fuertes. Sin embargo, cuando los niños también quieren expresar nociones que no están gramaticalizadas en su lengua, lo pueden intentar creando nuevas formas, categorías emergentes, que utilizarán durante algún tiempo. Como ejemplo, Clark (2001) propone la distinción entre propiedades inherentes-transitorias, una categoría emergente para los niños de habla inglesa pero una distinción gramaticalizada en los verbos ser y estar y, por tanto, una categoría fuerte para los aprendices del español.

Alternativamente, un creciente número de autores considera que el lenguaje puede influir en el desarrollo cognitivo. Por ejemplo, Gopnik, Choi y Baumberger (1996) demostraron que el aprendizaje de una lengua concreta (inglés o coreano) afecta de forma también concreta al momento de aparición de nociones, como la permanencia del objeto. El logro de esta noción coincidiría con el uso de expresiones lingüísticas que, precisamente, codifican la desaparición de objetos, como gone en inglés.

Frente a estas posiciones unidireccionales, se puede sostener una postura más interaccionista. Por ejemplo, Mueller Gathercole (2006) plantea que las características específicas de una lengua pueden facilitar o dificultar la adquisición de ciertas categorías gramaticales. Mientras que algunas nociones conceptuales se expresarían lingüísticamente a muy temprana edad, otras lo harían más tarde.

Este artículo se centra en el estudio de los verbos copulativos españoles ser y estar, que hacen diferente a esta lengua de otras muchas que cuentan con un único verbo copulativo (Fogsgaard, 2000). En español la distinción conceptual entre propiedades estables/transitorias se encuentra gramaticalizada en los verbos serlestar con adjetivos y, por tanto, supone un aprendizaje obligatorio para los niños que adquieren el español como lengua materna (Sera, 2008). Específicamente, en este estudio se pretendió abordar la influencia de ser y estar con adjetivos en las inferencias que hacen los hablantes de español sobre la estabilidad o transitoriedad de algunos rasgos psicológicos.

Los verbos copulativos tienen exclusivamente la función gramatical de unir al sujeto con el predicado, conectando el adjetivo con el sustantivo (Fernández, 1999). En español algunos ad- 
jetivos se combinan solo con una cópula, mientras que otros pueden hacerlo con ambas, dependiendo del significado y del contexto. Esta última posibilidad resulta especialmente compleja para los estudiosos del tema y para los aprendices del español como segunda lengua (GuijarroFuentes \& Geeslin, 2008). Hay abundante bibliografía lingüística sobre los verbos ser y estar, reflejo del interés suscitado por la dificultad de sistematizar la distribución de ambas cópulas (Falk, 1979; Holtheuer, 2011; Navas-Ruiz, 1977).

Se han propuesto distintas oposiciones para distinguir el uso de un adjetivo con uno u otro verbo, por ejemplo, las distinciones estable/transitorio o cualidades/estados, que hacen referencia a propiedades permanentes o temporales y, más recientemente, la distinción entre predicados individuales/de estadios. Mientras ser solo admite predicados individuales, que denotan propiedades de entidades al margen de cualquier localización espacio-temporal, estar solo admite predicados de estadios, que expresan propiedades transitorias respecto de una localización espacio-temporal (Fernández, 1999).

Paradójicamente, la abundante bibliografía en lingüística contrasta con las escasas publicaciones sobre la adquisición de ser y estar en aprendices nativos del español. Por lo que respecta a los estudios con habla espontánea, Sera (1992, Estudio 1) analizó los datos longitudinales del lenguaje de dos niños madrileños (desde los 18 meses hasta los 3,5 años, corpus de Linaza), así como las narraciones con el cuento de la rana de 46 niños (entre 3 y 11 años) y cinco adultos (corpus de E. Sebastián). En ambos casos, los participantes produjeron ambas cópulas con adjetivos, pero con uno u otro verbo. Posteriormente, Silva-Corvalán y Montanari (2008) analizaron el habla de un niño bilingüe (español-inglés) desde los 18 meses hasta los 3 años, encontrando que la distribución de ambos verbos muestra un sesgo que lleva a usar un determinado adjetivo con solo una de las cópulas.

En estudios experimentales Sera (1992, Estudio 4) constató en participantes cubano-americanos de 3,5 a 11,0 años la existencia de diferencias significativas debidas a la edad, aunque los niños menores usaron correctamente los verbos en el $70 \%$ de los casos. Por su parte, Herrera y Johnson (2005) hallaron diferencias significativas en una tarea de completar frases en niños mexicanos (2,5 a 6,3 años), cometiendo menos errores en las oraciones con estar.

Una perspectiva distinta se abre cuando se analizan los datos de comprensión. Schmitt, Holtheuer y Miller (2004) diseñaron dos tareas: una de emparejamiento de frases con una de las dos cópulas con dibujos de objetos con propiedades permanentes o transitorias y otra de evaluación de frases con ser/estar con adjetivos. En ambas tareas la actuación de los preescolares y de los adultos difirió significativamente, pues los niños no dominaron la distinción. En otro estudio con niños de 5 años, Schmitt y Miller (2007) presentaron una historia sobre un personaje con una característica permanente que sufría una transformación física —cambia de tamaño, color o peso- durante un breve periodo de tiempo. En este contexto, se debía evaluar la característica inherente del personaje —antes de la transformación temporal- y restringir la característica temporal a un periodo limitado de tiempo. Los resultados mostraron que, a diferencia de los adultos, los niños tenían dificultades para restringir el dominio temporal de ambos verbos, presentando problemas de tipo pragmático. Alonqueo y Soto (2005) realizaron un estudio con niños (5 a 12 años) y adultos madrileños mediante una tarea de emparejamiento de frases con adjetivos nuevos con objetos que tenían propiedades estables y transitorias, encontrando que solo los participantes de 12 años y los adultos proyectaban las propiedades estables en formas de ser con adjetivos y las transitorias con estar con adjetivos, por lo que las autoras concluyeron que la comprensión del contraste semántico ser/estar se consigue a partir de los 12 años. En resumen, los trabajos realizados muestran que los preescolares producen formas de serlestar con adjetivos desde edades tempranas, pero que la comprensión semántica de estas no se logra hasta la pre-adolescencia.

Si los trabajos sobre la adquisición de ser y estar son escasos, aún menor es la atención que ha recibido el estudio de sus posibles efectos en la cognición, pese a la relevancia conceptual de la dicotomía estable/transitorio. Entender que las entidades tienen propiedades permanentes o temporales es esencial para comprender las características del mundo físico y social y para formar conceptos (Gelman, Heyman \& Legare, 2007). Desde hace tiempo se sabe que los pre- 
escolares tienen dificultades para distinguir entre ambos tipos de propiedades. Por ejemplo, no dominan plenamente la distinción apariencia/realidad (Flavell, Flavell \& Green, 1987), no comprenden que el género es una característica estable (Eaton \& Von Bargen, 1981) ni que la identidad de un animal no se modifica por alteraciones aparentes (Keil, 1989).

Los aprendices del español contarían, sin embargo, con una herramienta lingüística que les ayudaría a distinguir entre los distintos tipos de propiedades, gracias al contraste ser/estar. Sera, Bales y Del Castillo Pintado (1997) realizaron un estudio en el que participaron niños monolingües españoles y monolingües ingleses de 3,1 a 5,8 años. En español las preguntas sobre propiedades reales se presentaron con ser y las sobre propiedades aparentes con estar, mientras que en inglés ambas preguntas se presentaron con el verbo to be. Los resultados mostraron que los niños de ambas lenguas identificaron fácilmente las propiedades aparentes, mientras que tuvieron más dificultades para reconocer las propiedades reales. Sin embargo, los niños españoles identificaron propiedades reales de objetos no familiares mejor que los niños hablantes de inglés. Según Sera et al. (1997), esta diferencia sugiere que el verbo ser proporciona información sobre lo que algo es en realidad.

Asimismo, para establecer el efecto de ser/estar en la categorización, Sera (1992, Estudio 2) realizó una investigación con adultos hablantes de español, quienes debieron aprender una nueva categoría en base a la descripción de las propiedades estables y transitorias de una entidad por medio de frases con serlestar con adjetivos. Los resultados mostraron que las propiedades descritas con el verbo ser fueron fundamentales para definir una categoría, mientras que las descritas con estar no proporcionaron información relevante. Alonqueo y Soto (2008) utilizaron una tarea semejante con niños y adultos hablantes de español, obteniendo resultados similares. Por tanto, pareciera que la distinción ser/estar facilita el proceso de formación de nuevas categorías a partir de los 12 años.

\section{Lenguaje e Inferencias Sobre Rasgos Psicológicos}

La relación entre el lenguaje - en especial la manera en que se habla sobre las personas-y las inferencias sobre rasgos psicológicos ha sido descrita en otros estudios (Diesendruck \& HaLevi, 2006; Yuill \& Pearson, 1998). Gelman y Heyman (1999) encontraron que los niños de cinco años eran sensibles a algunas claves lingüísticas, puesto que la descripción de una conducta seguida de una frase nominalizada en inglés llevaba a inferir que esta se mantendría estable en el tiempo (e.g., "Rosa es una come-zanahorias" frente a "Rosa, siempre que puede, come zanahorias"). La exposición a distinciones lingüísticas facilita que los niños puedan reconocer mejor sus implicaciones conceptuales y que exploten las posibilidades de su lengua para expresar sus creencias de manera más precisa (Shatz, Martínez-Beck, Diesendruck \& Akar, 2003). Así, las etiquetas lingüísticas funcionan como pistas que activan determinado tipo de suposiciones como, por ejemplo, el esencialismo. Aun cuando este concepto ha sido definido de diversas maneras (véase Gelman, 2003 y Medin \& Ortony, 1989 para una revisión), en general se acepta que la esencia de una entidad, aunque es de carácter subyacente y no observable, determina su identidad; ello implica suponer que un determinado rasgo puede ser estable e inmodificable.

Gelman (2003) señala — a partir de los datos de Sera (1992) — que ser/estar permite dar cuenta de las esencias de las entidades y, por tanto, podría ser útil para razonar sobre estas. Siguiendo esta línea argumental, Heyman y Diesendruck (2002) realizaron una serie de estudios con niños (6 a 10 años) bilingües inglés-español para establecer si describir características personales con los verbos ser, estar o to be influía en las inferencias sobre la estabilidad de estas. Se basaron en el supuesto de que una característica descrita con ser informa de su estabilidad, pero si es descrita con estar, da cuenta de su transitoriedad y posibilidad de cambio. Este efecto solo se observó en los niños mayores de 7,8 años, pues los menores consideraron que las carac- 
terísticas se mantendrían estables, independientemente del verbo utilizado para describirlas. Sin embargo, este estudio presenta algunos problemas que pueden afectar los resultados obtenidos. La diferencia entre las condiciones ser y estar es marginalmente significativa $(p>0,06)$ $y$, entonces, no se podría afirmar categóricamente que los escolares infieren que una característica presentada con ser será estable. Es posible que esta tendencia aumente con la edad e, incluso, los mismos autores subrayan que es necesario investigar estos cambios evolutivos en edades más amplias. Además, hubiera sido imprescindible contar con un grupo de comparación de adultos para determinar el nivel de los hablantes maduros. Otro aspecto crítico es que los participantes eran bilingües español-inglés residentes en Estados Unidos, teniendo como lengua materna variantes del español (español de México o de América Central). El "problema" de estas variantes es que en zonas de contacto con el inglés el uso de estar ha cambiado e, incluso, se ha extendido a contextos donde originalmente se usaba ser (Silva-Corvalán, 1986). Por último, los adjetivos seleccionados para describir las características psicológicas deberían poder usarse con ambos verbos sin cambiar su valor léxico, por ejemplo, es distraído, está distraído. Pero, ser listo equivaldría a ser inteligente, mientras que estar listo señala que se está preparado para hacer algo; por tanto, las inferencias que se hagan en uno y otro caso se referirán a características diferentes.

En el presente artículo se presentan dos estudios en los que participaron adultos (grupo de comparación) y niños monolingües del español de 7 y 12 años. El objetivo de esta investigación fue establecer si la distinción serlestar influye en las inferencias sobre la estabilidad de ciertas características psicológicas. Se formuló la hipótesis de que si los participantes se dejan guiar por la información proporcionada por ser y estar, las inferencias realizadas varían según el verbo utilizado para describir las características personales.

\section{Método}

\section{Participantes}

En ambos estudios se usó un muestreo no probabilístico por conveniencia, seleccionando a niños de escuelas públicas de la ciudad de Madrid y a adultos estudiantes de la Universidad Autónoma de Madrid. Todos ellos son hablantes de español de nivel socioeconómico medio.

En el Estudio 1 los participantes fueron 98, distribuidos en 32 escolares (16 niñas y 16 niños), entre 6,7 y 7,8 años $(M=7,3$ años, $D E=0,26)$ y 66 adultos (40 mujeres y 26 hombres) con una edad promedio de 22,1 años $(D E=0,45)$. Los participantes del Estudio 2 fueron 78, distribuidos en 30 escolares (mitad hombres y mujeres) entre 11,0 y 13,0 años $(M=11,9$ años, $D E=$ $0,41)$ y 42 adultos (28 mujeres y 14 hombres), cuya edad promedio fue 23,3 años $(D E=0,52)$.

\section{Instrumento}

En el Estudio 1 se utilizó una tarea de inferencias de elaboración ad-hoc, para lo cual se seleccionó un conjunto de adjetivos que en español peninsular se usaran indistintamente con serlestar y se solicitó a dos expertos en lingüística que evaluaran la gramaticalidad de las formas propuestas. Hubo un acuerdo de $100 \%$ entre los jueces con los adjetivos perezoso, cariñosa, inquieta, aburrida, alegre y despistado. Los tres últimos fueron seleccionados para construir dos versiones de la tarea (una con ser y la otra con estar), con tres historias en las que se describe la conducta de un escolar utilizando ser o estar con un adjetivo que codifica una característica psicológica, y a continuación se formularon cuatro preguntas. Este material fue probado con cinco adultos y 10 niños para verificar la inteligibilidad de las consignas. A partir de ello, solo se realizaron modificaciones en la extensión de las frases empleadas (Tabla 1). 
Tabla 1

Historia 1 del Cuestionario del Estudio1

En primer lugar, te presentaremos una breve historia sobre las características de una niña. A continuación, debes responder cuatro preguntas relacionadas con la historia que antes te presentamos.

Sara es una niña de 10 años. Sara es / está aburrida. Los domingos por la tarde se queda en casa y no sale.

1. ¿Por qué crees que Sara se queda en casa y no sale?

2. ¿Crees que en vacaciones Sara también se queda en casa sin salir?

3. ¿Crees que Sara seguirá quedándose en casa sin salir cuando sea mayor?

4. ¿Crees que Sara se quedaba en casa sin salir cuando tenía 8 años?

Para el Estudio 2 se modificaron algunas características de la tarea anterior. Además de los adjetivos aburrida y despistado (se eliminó alegre, pues en el Estudio 1 no hubo diferencias entre las condiciones experimentales), se incluyó inquieta, cariñosa y perezoso. Se usó un formato de respuesta dicotómica, pues en las preguntas de formato abierto del Estudio 1 los adultos proporcionaron información que iba más allá del contenido presentado en las historias, con lo cual resultaba difícil establecer el efecto de ser/estar en las inferencias. Con los adjetivos seleccionados se elaboraron cinco historias, diseñando dos versiones (una con ser y la otra con estar), respecto de las cuales se debía responder a dos preguntas (Tabla 2).

Tabla 2

Historia 1 del Cuestionario del Estudio 2

Sara tiene 20 años. Hoy ha ido al cumpleaños de su vecina, se ha sentado sola y no ha hablado con los otros invitados. Sara es/está muy aburrida.

De acuerdo con esta historia, es probable que:

1.1. Sara también se sienta sola y no hable con otras personas cuando vaya a otra fiesta SÍ NO

1.2. Sara a los 25 años también se sienta sola y no hable con otras personas cuando vaya a una $\quad$ Sí NO fiesta

\section{Procedimiento}

En ambos estudios se informó a los participantes de los objetivos de la investigación, el anonimato y la confidencialidad de los datos. Esta información se presentó en un formulario de consentimiento informado, que fue firmado por los adultos antes de la aplicación de la tarea. En el caso de los niños, los padres firmaron dicho documento, luego de que estos les manifestaran verbalmente su acuerdo con participar en el estudio.

Los participantes fueron asignados aleatoriamente a una de dos condiciones experimentales - condición ser y condición estar - que diferían únicamente en el verbo empleado para describir las características de los personajes.

Los adultos respondieron a una versión colectiva de la tarea que duró aproximadamente 15 minutos. Una experimentadora aplicó la tarea individualmente a los niños durante un periodo aproximado de 20 minutos. 


\section{Análisis de la Información}

En el Estudio 1 las respuestas de las tres historias se codificaron según dos categorías: uso de los verbos serlestar para explicar la conducta de los personajes (pregunta 1) e inferencias sobre la estabilidad o transitoriedad de las conductas asociadas a los rasgos evaluados (preguntas 2,3 y 4 ).

El análisis de las respuestas a la pregunta 1 mostró que la información contenida en el contraste serlestar no fue usada para explicar la conducta del protagonista (la respuesta esperada hubiese sido una afirmación como "Porque es [o está] despistado"), de modo que estos datos no fueron útiles para evaluar el uso de ambos verbos y se excluyeron del análisis. Tanto niños como adultos se centraron en buscar explicaciones extralingüísticas. Por ejemplo, en la historia "Sara es una niña de 10 años. Sara es aburrida. Los domingos por la tarde se queda en casa y no sale” y frente a la pregunta “¿Crees que María seguirá jugando mucho con otros niños y niñas cuando sea mayor?", un niño responde: "No, saldrá con sus amigas. A lo mejor de mayor ha aprendido que tiene que divertirse porque si se queda en casa aburrida no sabe qué hacer" (participante 10, 7,3 años). En los adultos ocurrió algo similar. Por ejemplo, en la historia "Pablo es un niño de 9 años. Pablo es despistado. Ha olvidado el libro de mates en casa" y frente a la pregunta "¿Crees que Pablo seguirá olvidando cosas en casa cuando sea mayor?" un participante responde "que sea despistado no significa que siempre le pase lo mismo" (participante 31). Las respuestas a las preguntas que evaluaban inferencias — nueve en total, tres en cada ensayo- se puntuaron asignando un valor 0 , si se respondía negativamente, o 1 , si respondía afirmativamente. Se calculó la medida inferencias sumando las puntuaciones de las tres historias, la cual podía oscilar entre 0 y 9 puntos. Una puntuación 0 significa que se infiere que todas las características son transitorias, presentando un bajo grado de estabilidad y, por tanto, no se generalizan en el tiempo ni a otros contextos, mientras que una puntuación 9 significa que las características presentan un alto grado de estabilidad y, por tanto, son generalizables. Del mismo modo, se tabularon las respuestas en el Estudio 2 con la diferencia de que las puntuaciones podían oscilar entre 0 y 10 puntos.

En ambos estudios, mediante una serie de pruebas $t$ para una muestra, se realizó un análisis estadístico para determinar si las respuestas de los participantes fueron influidas por el azar. Se realizó un ANOVA factorial mixto o de medidas repetidas: 3 (Característica) x 2 (Condición) x 2 (Edad), con Característica como factor intrasujetos y Condición y Edad como factores intersujetos que, en el caso del Estudio 2, correspondió a 5 (Característica) x 2 (Edad) x 2 (Condición). El análisis de los efectos simples se realizó mediante una serie de pruebas $t$ con la corrección de Bonferroni y permitió comparar las puntuaciones en cada condición experimental al interior de los dos grupos de edad.

\section{Resultados}

\section{Estudio 1}

En la Tabla 3 se muestran las puntuaciones medias en inferencias en niños y adultos en ambas condiciones. El análisis mostró que en la condición ser la actuación de los niños, $t(15)=$ $3,18, p=0,006, d=0,79,95 \%$ IC [0,41, 2,08], y de los adultos, $t(32)=3,75, p=0,001, d=0,65$, $95 \%$ IC $[0,31,1,05]$, difirió de la esperada por azar, al igual que los resultados obtenidos en la condición estar en niños, $t(15)=4,15, p=0,001, d=1,13,95 \%$ IC $[0,82,2,30]$, y adultos, $t(32)=$ $-8,89, p=0,001, d=-1,56,95 \%$ IC $[-1,84,-1,15]$. 
Tabla 3

Estudio 1: Puntuaciones Medias en Inferencias, Según Edad y Condición

\begin{tabular}{|c|c|c|c|c|c|c|}
\hline \multirow{3}{*}{ Condición } & \multicolumn{5}{|c|}{ Característica } & \multirow{3}{*}{$N$} \\
\hline & $\begin{array}{c}\text { Aburrida } \\
(0-3)\end{array}$ & $\begin{array}{c}\text { Alegre } \\
(0-3)\end{array}$ & $\begin{array}{c}\text { Despistado } \\
(0-3)\end{array}$ & $\begin{array}{c}\text { Promedio } \\
\quad(0-3)\end{array}$ & $\begin{array}{l}\text { Total } \\
(0-9)\end{array}$ & \\
\hline & $M(D E)$ & $M(D E)$ & $M(D E)$ & $M(D E)$ & $M(D E)$ & \\
\hline \multicolumn{7}{|l|}{ Condición ser } \\
\hline Niños & $1,0(0,96)$ & $2,9(0,34)$ & $1,9(0,88)$ & $1,9(0,09)$ & $5,8(1,57)$ & 16 \\
\hline Adultos & $1,3(0,62)$ & $2,6(0,48)$ & $1,3(0,62)$ & $1,7(0,06)$ & $5,2(1,04)$ & 33 \\
\hline Total & $1,2(0,75)$ & $2,7(0,45)$ & $1,5(0,76)$ & $1,8(0,06)$ & $5,4(1,25)$ & 49 \\
\hline \multicolumn{7}{|l|}{ Condición estar } \\
\hline Niños & $1,3(0,85)$ & $2,8(0,57)$ & $2,1(0,68)$ & $2,0(0,09)$ & $6,1(1,38)$ & 16 \\
\hline Adultos & $0,5(0,66)$ & $1,8(0,84)$ & $0,7(0,76)$ & $1,0(0,06)$ & $3,0(0,96)$ & 33 \\
\hline Total & $0,7(0,81)$ & $2,1(0,86)$ & $1,2(0,76)$ & $1,5(0,06)$ & $4,0(1,82)$ & 49 \\
\hline \multicolumn{7}{|c|}{ Ambas condiciones } \\
\hline Niños & $1,1(0,13)$ & $2,8(0,11)$ & $2,0(0,12)$ & $2,0(0,07)$ & $5,9(1,82)$ & 32 \\
\hline Adultos & $0,8(0,09)$ & $2,2(0,07)$ & $1,0(0,09)$ & $1,4(0,05)$ & $4,1(1,49)$ & 66 \\
\hline Total & $1,0(0,08)$ & $2,5(0,07)$ & $1,5(0,08)$ & $1,7(0,05)$ & $4,7(1,70)$ & 98 \\
\hline
\end{tabular}

Se observó un efecto principal del factor Característica, considerando las puntuaciones obtenidas en aburrida, alegre y despistado, $F(2,94)=103,29, p=0,001, \eta_{p}^{2}=0,52$, ICs $95 \%[0,84$, $1,15],[2,39,2,66]$ y $[1,331,64]$, respectivamente. El efecto del factor Condición también fue significativo, teniendo en cuenta las puntuaciones medias observadas en la condiciones ser y estar, $F(1,94)=13,50, p=0,001, \eta_{p}^{2}=0,13$, ICs $95 \%[1,70,1,94]$ y $[1,39,1,62]$, respectivamente. En el factor Edad la puntuación media de los niños difirió de la obtenida por los adultos, de modo que el efecto resultó significativo, $F(1,94)=51,02, p=0,001, \eta_{p}^{2}=0,35$, ICs $95 \%[1,83,2,11] \mathrm{y}$ $[1,27,1,46]$, respectivamente.

Hubo efectos interactivos estadísticamente significativos entre Condición y Edad, considerando las puntuaciones medias de niños y adultos en ambas condiciones experimentales (ser y estar), $F(1,94)=24,08, p=0,001, \eta_{p}^{2}=0,20$, ICs $95 \%[1,72,2,11],[1,82,2,21],[1,59,1,86]$ y $[0,86,1,13]$, respectivamente. El análisis de los efectos simples mostró que las diferencias solo se produjeron en los adultos (Figura 1), pues el grado de estabilidad de las inferencias en la condición ser difirió significativamente de la condición estar, $t(64)=8,80, p=0,001, d=$ $2,23,95 \%$ IC [1,68, 2,67]. El verbo ser permitió inferir que las conductas asociadas a los rasgos psicológicos se mantendrían estables, mientras que estar indicó que dichas conductas eran transitorias y, por ende, no las generalizaron. Los niños registraron puntuaciones similares en ambas condiciones, no observándose diferencias estadísticamente significativas, $t(30)=-0,60$, $p=0,456$. Resulta llamativo que estas puntuaciones sean altas, lo cual podría indicar la existencia de algún sesgo hacia una visión estable y permanente de los rasgos. 


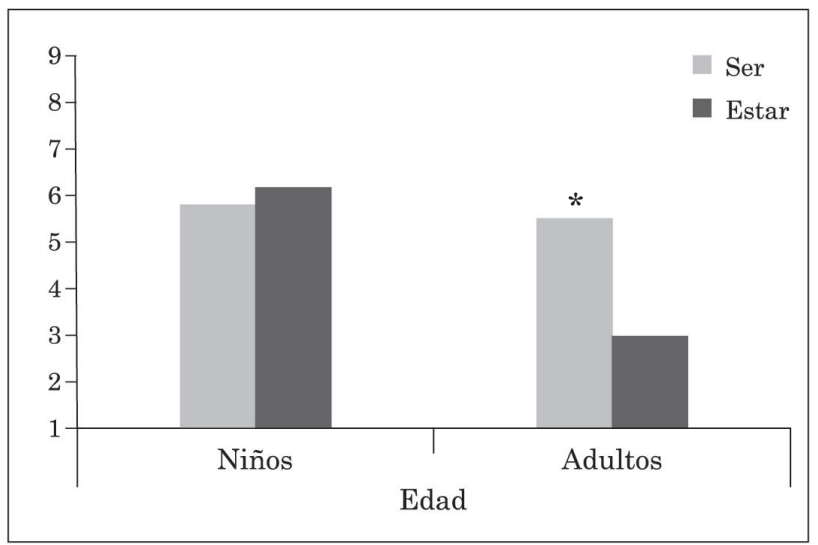

Figura 1. Puntuación media en inferencias, según edad y condición. ${ }^{*} p<0,001$.

También el análisis mostró efectos interactivos entre Característica y Edad (ver promedios en la Tabla 3), $F(2,94)=5,34, p=0,006, \eta_{p}^{2}=0,05$, ICs $95 \%[0,86,1,38],[2,59,3,03]$ y $[1,71$, $2,22]$ en niños y $[0,68,1,04],[2,07,2,38]$ y $[0,82,1,17]$ en adultos, en aburrida, alegre y despista$d o$, respectivamente. La serie de pruebas $t$ con la corrección de Bonferroni mostró que, aunque para ambos grupos (Figura 2) alegre es la característica que se considera más estable — seguida por despistado y aburrida - la puntuación de los niños en alegre es significativamente mayor que la de los adultos, $t(96)=3,82, p=0,001, d=7,10,95 \%$ ICs $[2,59,3,03]$ y $[2,07,2,38]$, respectivamente, al igual que las puntuaciones en despistado, $t(96)=5,94, p=0,001, d=9,75,95 \%$ ICs $[1,71,2,22]$ y $[0,82,1,18]$, respectivamente. En aburrida las diferencias no fueron estadísticamente significativas, $t(96)=1,50, p=0,138$.

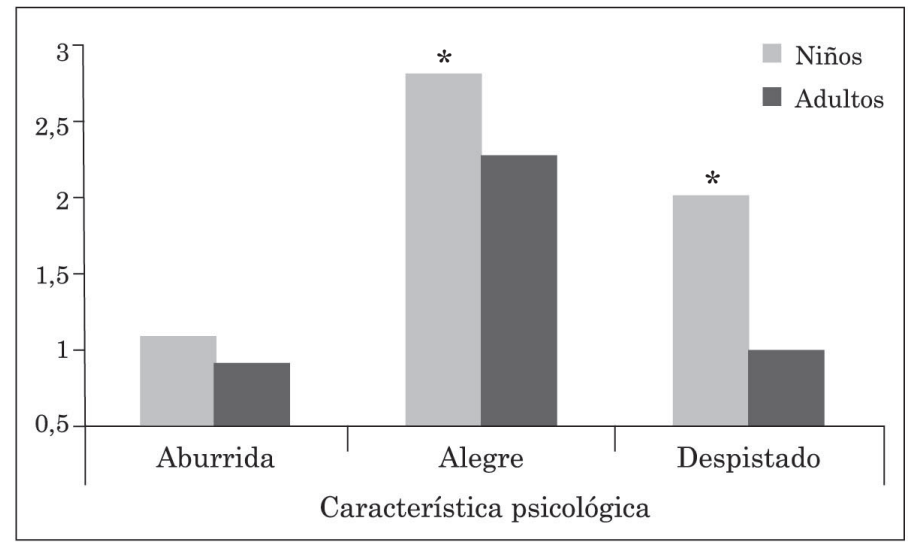

Figura 2. Puntuación media en característica psicológica, según edad. * $p<0,001$.

No se observaron efectos interactivos entre Característica y Condición, $F(2,94)=094, p=$ 0,391, ni entre Característica, Condición y Edad, $F(2,94)=0,45, p=0,637$.

En suma, estos resultados permiten afirmar que la influencia de la distinción ser/estar en las inferencias solo se observa en el grupo de adultos. La información proporcionada por estos verbos promueve inferencias completamente distintas cuando se razona sobre la estabilidad 
de los rasgos psicológicos. Mientras que presentar determinados rasgos con ser permite inferir que estos se mantendrán estables a lo largo del tiempo y en diferentes contextos, presentar esos mismos rasgos con estar permite inferir que dichas características son transitorias. Los escolares, por su parte, no utilizaron la distinción lingüística ser/estar para razonar sobre los rasgos evaluados en este estudio.

Si se analizan por separado los rasgos presentados con ser, se aprecia que estos no se perciben con el mismo grado de estabilidad (véase la Tabla 3). Por ejemplo, alegre se percibe más estable que los otros dos rasgos. Estos hallazgos sugieren que es posible que en las inferencias sobre la estabilidad de las características no solo influya el uso de ser o estar, sino también, el tipo de rasgo evaluado y las creencias que se tengan sobre su estabilidad.

\section{Estudio 2}

En consideración a que los escolares participantes en el Estudio 1 no fueron sensibles a la influencia de serlestar al razonar sobre rasgos psicológicos, se realizó un segundo estudio incorporando una mayor cantidad de características e incluyendo a un grupo de 12 años, ya que estudios previos (Alonqueo \& Soto, 2005) han mostrado que a esa edad se comienza a comprender la relación entre ser/estar con adjetivo y propiedades estables/transitorias, y que dicha información se usa como base para formar categorías. Las hipótesis para este estudio fueron: a) ambos grupos de edad muestran un patrón de actuación similar: en la condición ser se infiere estabilidad y universalidad y en la condición estar se infiere transitoriedad de la conducta y b) el grado de estabilidad con el que se perciben las características psicológicas varía según el rasgo evaluado.

Se compararon las puntuaciones de los participantes con la puntuación esperada por azar (ver los promedios en la Tabla 4), obteniendo que en la condición ser no hubo diferencias significativas en el caso de los niños, $t(15)=0,69, p=0,505$, ni de los adultos, $t(20)=0,68, p=0,504$, al contrario de los resultados obtenidos en la condición estar por niños, $t(14)=-2,58, p=0,022$, $d=-0,69,95 \%$ IC $[3,25,5,01]$, y adultos, $t(14)=6,85, p=0,001, d=-0,80,95 \%$ IC $[2,78,4,26]$.

Tabla 4

Estudio 2: Puntuaciones Medias en Inferencias, Según Edad y Condición

\begin{tabular}{|c|c|c|c|c|c|c|c|c|}
\hline \multirow{3}{*}{ Condición } & \multicolumn{7}{|c|}{ Característica } & \multirow{3}{*}{$N$} \\
\hline & $\begin{array}{c}\text { Aburrida } \\
\quad(0-2)\end{array}$ & $\begin{array}{c}\text { Despistado } \\
(0-2)\end{array}$ & $\begin{array}{l}\text { Inquieta } \\
(0-2)\end{array}$ & $\begin{array}{l}\text { Cariñosa } \\
\quad(0-2)\end{array}$ & $\begin{array}{l}\text { Perezoso } \\
(0-2)\end{array}$ & $\begin{array}{c}\text { Promedio } \\
(0-2)\end{array}$ & $\begin{array}{l}\text { Total } \\
(0-10)\end{array}$ & \\
\hline & $M(D E)$ & $M(D E)$ & $M(D E)$ & $M(D E)$ & $M(D E)$ & $M(D E)$ & $M(D E)$ & \\
\hline \multicolumn{9}{|l|}{ Condición ser } \\
\hline Niños & $0,9(0,88)$ & $1,1(0,59)$ & $1,1(0,79)$ & $1,3(0,75)$ & $1,0(0,75)$ & $1,0(0,08)$ & $5,4(2,26)$ & 15 \\
\hline Adultos & $1,0(0,89)$ & $1,0(0,83)$ & $1,2(0,88)$ & $1,1(0,80)$ & $1,1(0,80)$ & $1,1(0,12)$ & $5,3(2,24)$ & 21 \\
\hline Total & $1,0(0,34)$ & $1,0(0,11)$ & $1,2(0,13)$ & $1,2(0,20)$ & $1,0(0,11)$ & $1,1(0,07)$ & $5,4(2,21)$ & 36 \\
\hline \multicolumn{9}{|l|}{ Condición estar } \\
\hline Niños & $0,3(0,48)$ & $1,1(0,74)$ & $0,9(0,63)$ & $1,1(0,74)$ & $0,7(0,48)$ & $0,8(0,10)$ & $4,1(1,30)$ & 15 \\
\hline Adultos & $0,7(0,90)$ & $0,4(0,58)$ & $0,9(0,72)$ & $1,2(0,67)$ & $0,4(0,49)$ & $0,7(0,08)$ & $3,5(1,88)$ & 21 \\
\hline Total & $0,4(0,14)$ & $\mathrm{O}, 8(0,11)$ & $0,9(0,13)$ & $1,2(0,12)$ & $0,5(1,11)$ & $0,8(0,07)$ & $3,4(1,67)$ & 36 \\
\hline \multicolumn{9}{|c|}{ Ambas condiciones } \\
\hline Niños & $0,6(0,12)$ & $1,1(0,12)$ & $0,9(0,14)$ & $1,2(0,13)$ & $0,8(0,12)$ & $0,9(0,07)$ & $4,4(2,24)$ & 30 \\
\hline Adultos & $0,8(0,12)$ & $0,7(0,10)$ & $1,0(0,12)$ & $1,1(0,10)$ & $0,7(0,10)$ & $0,9(0,06)$ & $4,8(1,92)$ & 42 \\
\hline Total & $0,7(0,09)$ & $0,9(0,08)$ & $1,0(0,09)$ & $1,2(0,09)$ & $0,8(0,07)$ & $0,9(0,04)$ & $4,6(2,10)$ & 72 \\
\hline
\end{tabular}


El análisis mostró un efecto significativo del factor Característica, pues las diferencias entre las puntuaciones medias obtenidas en cada característica fueron estadísticamente significativas, $F(4,68)=10,54, p=0,002, \eta_{p}^{2}=0,061$, ICs 95\% [0,54, 0,94], [0,72, 1,06], [0,82, 1,19], [0,99, $1,35]$ y $[0,61,0,93]$ para aburrida, despistado, inquieta, cariñosa y perezoso, respectivamente. También se obtuvo un efecto del factor Condición, pues las diferencias entre las condiciones ser y estar resultaron estadísticamente significativas, $F(1,68)=4,39, p=0,003, \eta_{p}^{2}=0,134$, ICs $95 \%[0,94,1,20]$ y $[0,63,0,89]$, respectivamente.

No hubo un efecto significativo del factor $\operatorname{Edad,~} F(1,68)=0,51, p=0,478$, ni tampoco interacciones estadísticamente significativas entre los factores Edad y Condición, $F(1,68)=0,328$, $p=0,569$, Edad y Característica, $F(4,68)=2,00, p=0,101$, Característica y Condición, $F(4,68)$ $=1,174, p=0,322$, y Edad, Característica y Condición, $F(4,68)=1,68, p=0,154$. Tales resultados indican que no hay efecto de la variable edad y que, como se predijo, los participantes de 12 años utilizaron la información proporcionada por serlestar de igual manera que los adultos (véase la Figura 3).

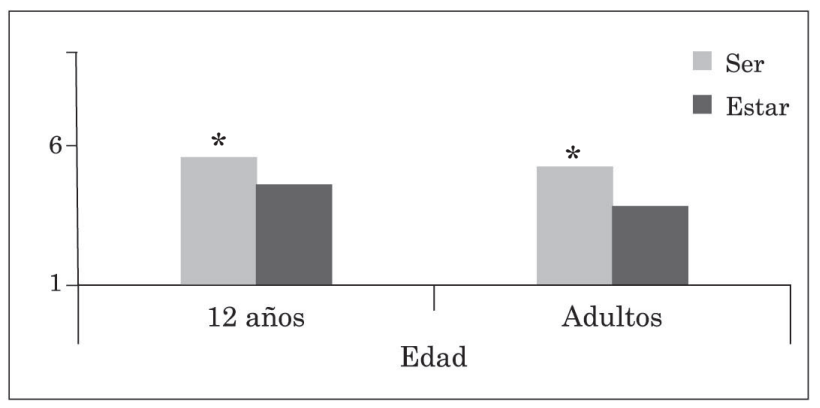

Figura 3. Puntuación media en inferencias, según edad y condición. ${ }^{*} p=0,003$.

Aunque en la Figura 4 se observa la tendencia a que los rasgos mencionados con ser se generalizan más que los presentados con estar, hay características, como cariñosa, que se perciben de manera estable en ambas condiciones experimentales. Los resultados de las pruebas $t$ con la corrección de Bonferroni muestran que solo las diferencias en aburrida, $t(70)=2,14, p=$ $0,036, d=0,55$, IC $95 \%[0,02,0,85]$, y en perezoso fueron estadísticamente significativas, $t(70)$ $=3,42, p=0,001, d=0,78, \mathrm{IC} 95 \%[0,89,1,23]$.

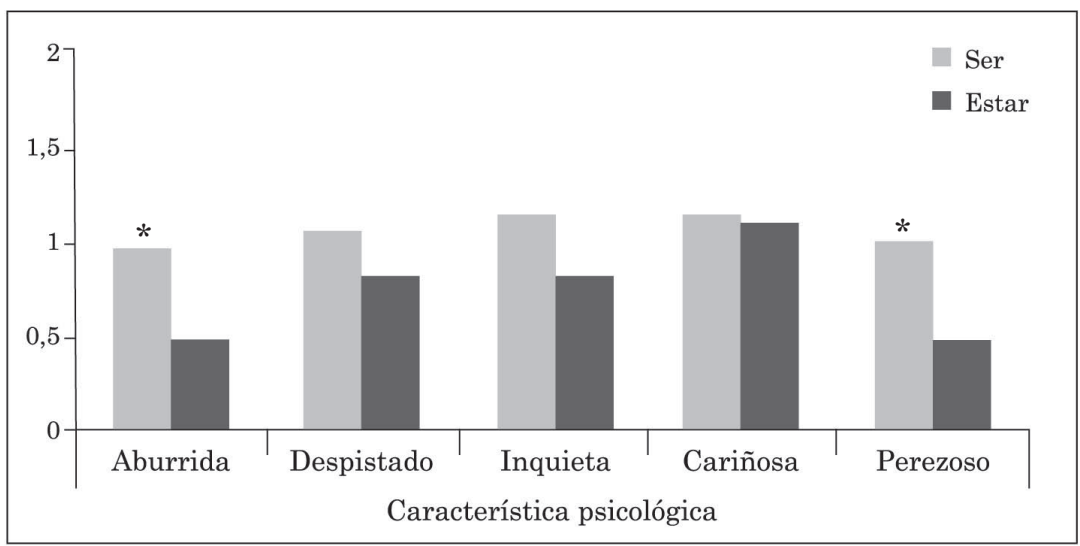

Figura 4. Puntuación media en características psicológicas, según condición. ${ }^{*} p \leq 0,003$. 
Por el contrario, en despistado no hubo diferencias estadísticamente significativas entre ambas condiciones experimentales, $t(70)=1,90, p=0,061$, al igual que en inquieta, $t(70)=1,69$, $p=0,096$.

En resumen, los datos obtenidos en este segundo estudio estarían indicando que el efecto de serlestar en las inferencias se observa a partir de la pre-adolescencia.

\section{Discusión}

El examen conjunto de los resultados de ambos estudios permite plantear algunas conclusiones importantes respecto de la influencia de serlestar en las inferencias y sus variaciones evolutivas.

En primer lugar, los datos obtenidos en el Estudio 2, a diferencia de los resultados del Estudio 1, muestran que no existen diferencias estadísticamente significativas según la edad de los participantes, pues la actuación del grupo de 12 años es más semejante a la de los adultos que a la de los niños de 7 años, quienes —independientemente de la información lingüística- presentan una tendencia a percibir las características psicológicas de manera estable.

Los datos de este estudio son similares a los obtenidos por Heyman y Diesendruck (2002) con niños cubano-americanos bilingües menores de 7 años. Los autores plantean que la escasa influencia de serlestar se debería a que los niños conciben los rasgos de manera esencialista y que, conforme avanza el desarrollo, esta tendencia disminuye (Bales \& Sera, 1995). En el presente estudio la actuación del grupo de 12 años podría interpretarse en ese mismo sentido, pero para ello sería necesario investigar todos los supuestos del esencialismo, cuestión que no ha sido el objetivo de esta investigación.

Si bien el lenguaje proporciona claves importantes para los conceptos, no necesariamente es el mecanismo primario a través del cual estos emergen (Gelman \& Kalish, 2006; Taverna $\&$ Peralta, 2009). Es necesaria una comprensión inicial de los conceptos para que las etiquetas lingüísticas proporcionen información relevante sobre la estructura de los mismos.

En la misma línea, existen datos sobre las diferencias con que preescolares y escolares perciben los rasgos psicológicos y las inferencias que realizan en base a estos. Bales y Sera (1995) concluyeron que comprender distintos tipos de características presenta diferente grado de dificultad. Las que suponen un cambio a largo plazo, como el peso y la edad, fueron más difíciles de comprender; en cambio, una característica transitoria, como el estado de ánimo, fue comprendida con mayor facilidad. Según las autoras, es posible que la comprensión de la dicotomía estable/transitorio, aplicada a las características personales, no se desarrolle a la manera todo o nada, sino que incluso se adquiera atributo por atributo. También es probable que los rasgos no sean, necesariamente, una fuente importante de inferencias, en comparación, por ejemplo, con las categorías naturales (Heyman \& Gelman, 1999). No obstante, por el momento, solo se puede proponer que los datos de los niños mayores parecen concordantes con la idea de que un modelo más complejo de los rasgos psicológicos comienza a surgir en la niñez intermedia (Gelman et al., 2007). Nuevos estudios deberían profundizar en este ámbito, superando las posibles limitaciones metodológicas derivadas del uso de cuestionarios de respuesta cerrada, planteando preguntas sobre los rasgos en sentido positivo y negativo.

La escasa influencia de ser/estar en las inferencias se podría explicar porque a lo largo de la edad escolar la relación entre lenguaje y cognición continúa desarrollándose (Lucy, 2005) y, por ello, los escolares aún no comprenden que dicha distinción lingüística marca la estabilidad y la transitoriedad de las propiedades. Esta comprensión se alcanzaría en la pre-adolescencia, facilitando procesos como el de categorización de objetos (Alonqueo \& Soto, 2005, 2008). Ello sería posible porque la adquisición de las formas lingüísticas supone, finalmente, la comprensión de las formas semánticas que le subyacen; a su vez, son estas las que ayudan a resaltar ciertos aspectos de la cognición, según lo que se codifique en una determinada lengua (Mueller Gathercole, 2006).

La actuación del grupo de 12 años y los adultos confirma el efecto de ser/estar en las inferencias descrito por Heyman y Diesendruck (2002). Como ya se planteó, los hablantes de español cuentan con una herramienta lingüística — contenida en la distinción ser y estar-que 
les permitiría concebir los rasgos psicológicos de manera menos permanente. Ello puede constituir una ventaja importante respecto de los angloparlantes, pues una visión más flexible de las características personales permitiría construir una teoría según la cual se suponga que las conductas asociadas a estas son modificables, cuestión altamente relevante, por ejemplo, para facilitar las relaciones intra e intergrupales (Estrada, Oyarzún \& Yzerbyt, 2007) o concebir la pobreza como un estado modificable (Del Río \& Strasser, 2007).

Aunque ser ayude a razonar sobre la estabilidad de los rasgos y estar facilite la comprensión sobre su transitoriedad, también las características en sí mismas tienen un papel importante. Claramente se aprecia que los rasgos no son afectados de igual manera por el uso de ser/estar; solo en el caso de aburrida y perezoso esta distinción marca una diferencia importante. Es probable que diferentes características se conciban con diferente grado de estabilidad (Heyman \& Gelman, 2000). Así, las variaciones entre los distintos rasgos se podrían explicar también en base a las teorías y creencias sobre qué características son modificables y cuáles no, independientemente de las expresiones lingüísticas utilizadas para referirse a ellas. Por tanto, el efecto del lenguaje en el razonamiento sobre rasgos psicológicos posiblemente esté matizado por la influencia de factores extralingüísticos, incluidos los cognitivos y los culturales.

\section{Referencias}

Alonqueo, P. \& Soto, P. (2005). La distinción ser/estar y la comprensión de propiedades estables y transitorias. En M. A. Mayor, B. Zubiauz \& E. Diez-Villoria (Eds.), Estudios sobre la adquisición del lenguaje (pp. 281-299). Salamanca, España: Ediciones Universidad de Salamanca.

Alonqueo, P. \& Soto, P. (2008). La influencia de ser/estar en la categorización en niños y adultos hablantes de español. En E. Diez-Itza (Ed.), Estudios de desarrollo del lenguaje y educación (pp. 105-114). Oviedo, España: Universidad de Oviedo, Instituto de Ciencias de la Educación.

Bales, D. W. \& Sera, M. D. (1995). Preschoolers' understanding of stable and changeable characteristics. Cognitive Development, 10, 69-107. doi:10.1016/0885-2014(95)90019-5

Bowerman, M. (1996). Learning how to structure space for language: A crosslinguistic perspective. En P. Bloom, M. A. Peterson, L. Nadel \& M. F. Garrett (Eds.), Language and space (pp. 385-436). Cambridge, MA: Massachusetts Institute of Technology.

Bowerman, M. \& Levinson, S. C. (Eds.) (2001). Language acquisition and conceptual development. Cambridge, Reino Unido: Cambridge University Press.

Clark, E. V. (2001). Emergent categories in first language acquisition. En M. Bowerman \& S. C. Levinson (Eds.), Language acquisition and conceptual development (pp. 379-405). Cambridge, Reino Unido: Cambridge University Press.

Dasen, P. R. \& Mishra, R. C. (2010). Development of geocentric spatial language and cognition: An ecocultural perspective. New York, NY: Cambridge University Press.

Del Río, M. F. \& Strasser, K. (2007). ¿Tienen los niños una teoría esencialista acerca de la pobreza? Psykhe, 16(2), 139149. doi:10.4067/S0718-22282007000200012

Diesendruck, G. \& HaLevi, H. (2006). The role of language, appearance, and culture in children's social category-based induction. Child Development, 77, 539-553. doi:10.1111/j.1467-8624.2006.00889.x

Eaton, W. \& Von Bargen, D. (1981). Asynchronous development of gender understanding in preschool children. Child Development, 52, 1020-1027.

Estrada, C., Oyarzún, M. \& Yzerbyt, V. (2007). Teorías implícitas y esencialismo psicológico: herramientas conceptuales para el estudio de las relaciones entre y dentro de los grupos. Psykhe, 16(1), 111-121. doi:10.4067/S071822282011000100005

Falk, J. (1979). Ser y estar con atributos adjetivales. Uppsala, Suecia: Uppsala Universitet, Acta Universitatis Uppsaliensis.

Fernández, M. J. (1999). La predicación: las oraciones copulativas. En I. Bosque \& V. Demonte (Eds.), Gramática descriptiva de la lengua española (Vol. 2, pp. 2357-2460). Madrid, España: Espasa Calpe.

Flavell, J. H., Flavell, E. R. \& Green, F. L. (1987). Young children's knowledge about the apparent-real and pretendreal distinctions. Developmental Psychology, 23, 816-822. doi:10.1037/0012-1649.23.6.81

Fogsgaard, L. (2000). Esquemas copulativos de SER y ESTAR: ensayo de semiolingüística. Bern, Suiza: Peter Lang.

Gelman, S. A. (2003). The essential child: Origins of essentialism in everyday thought. New York, NY: Oxford University Press.

Gelman, S. A. \& Heyman, G. D. (1999). Carrot-eaters and creature-believers: The effects of lexicalization on children's inferences about social categories. Psychological Science, 10, 489-493. doi:10.1111/1467-9280.00194

Gelman, S. A., Heyman, G. D. \& Legare, C. H. (2007). Developmental changes in the coherence of the essentialist beliefs about psychological characteristics. Child Development, 78, 757-774. doi:10.1111/j.1467-8624.2007.01031.x

Gelman, S. A. \& Kalish, C. W. (2006). Conceptual development. En D. Kuhn \& R. Sieglers (Eds.), Handbook of child psychology (Vol. 2: Cognition, perception and language, $6^{a}$ ed., pp. 688-733). New York, NY: John Wiley \& Sons.

Gentner, D. \& Goldin-Meadow, S. (2003). Language in mind: Advances in the study of language and thought. Cambridge, MA: Massachusetts Institute of Technology. 
Gopnik, A., Choi, S. \& Baumberger, T. (1996). Cross-linguistics differences in early semantic and cognitive development. Cognitive Development, 11, 197-227. doi:10.1016/S0885-2014(96)90003-9

Guijarro-Fuentes, P. \& Geeslin, K. L. (2008). Introduction to language acquisition, bilingualism and copula choice in Spanish. Bilingualism: Language and Cognition, 11, 273-275. doi:10.1017/S1366728908003507

Guo, J., Lieven, E., Budwig, N., Ervin-Tripp, S., Nakamura, K. \& Ozcaliskan, S. (Eds.) (2009). Crosslinguistic approaches to the psychology of language: Research in the traditions of Dan Isaac Slobin. Philadelphia, PA: Psychology Press.

Herrera, T. \& Johnson, C. M. (2005). Desarrollo lingüístico infantil: comprensión y uso de ser y estar en niños mexicanos. En M. A. Mayor, B. Zubiauz \& E. Diez-Villoria (Eds.), Estudios sobre la adquisición del lenguaje (pp. 204-210). Salamanca, España: Ediciones Universidad de Salamanca.

Heyman, G. D. \& Diesendruck, G. (2002). The Spanish ser/estar distinction in bilingual children's reasoning about human psychological characteristics. Developmental Psychology, 38, 407-417. doi:10.1037/0012-1649.38.3.407

Heyman, G. D. \& Gelman, S. A. (1999). The use of trait labels in making psychological inferences. Child Development, 70, 604-619. doi:10.1111/1467-8624.00044

Heyman, G. D. \& Gelman, S. A. (2000). Beliefs about the origins of human psychological traits. Developmental Psychology, 36, 663-678. doi:10.1037/0012-1649.36.5.663

Holtheuer, C. (2011). The distribution of the ser and estar with adjectives: A critical survey. Revista Signos, 44, 33-47. doi:10.4067/S0718-09342011000100003

Keil, F. C. (1989). Concepts, kinds, and cognitive development. Cambridge, MA: Massachusetts Institute of Technology.

Lucy, J. A. (1992). Grammatical categories and cognition: A case study of the linguistic relativity hypothesis. Cambridge, Reino Unido: Cambridge University Press.

Lucy, J. A. (2005). Through the window of language: Assessing the influence of language diversity on thought. Theoria, 20, 299-309.

Lucy, J. A. \& Gaskins, S. (2003). Interaction of language type and referent type in the development of nonverbal classification preferences. En D. Gentner \& S. Goldin-Meadow (Eds.), Language in mind: Advances in the study of language and thought (pp. 465-492). Cambridge, MA: Massachusetts Institute of Technology.

Medin, D. \& Ortony, A. (1989). Psychological essentialism. En S. Vosniadou \& A. Ortony (Eds.), Similarity and analogical reasoning (pp. 179-195). New York, NY: Cambridge University Press.

Mueller Gathercole, V. C. (2006). Introduction to special issue: Language specific influences on acquisition and cognition. First Language, 26, 5-17. doi:10.1177/0142723706060738

Navas-Ruiz, F. (1977). Ser y estar: el sistema atributivo del español. Salamanca, España: Almar.

Sapir, E. (1949). Selected writings in language, culture, and personality. Editado por D. G. Mandelbaum. Berkeley, CA: University of California Press.

Schmitt, C., Holtheuer, C. \& Miller, K. (2004). Acquisition of copulas ser and estar in Spanish: Learning lexicosemantics, syntax and discourse. University Park, PA: Pennsylvania State University, Language Acquisition Lab. Extraído de http://childlanguagelab.la.psu.edu/publications/schmitt.pdf

Schmitt, C. \& Miller, K. (2007). Making discourse-dependent decisions: The case of the copulas ser and estar in Spanish. Lingua, 117, 1907-1929. doi:10.1016/j.lingua.2006.11.007

Sera, M. D. (1992). To be or to be: Use and acquisition of the Spanish copulas. Journal of Memory and Language, 31, 408-427. doi:10.1016/0749-596X(92)90021-O

Sera, M. D. (2008). Commentary on "copular acquisition" - a response to Silva-Corvalán and Montari. Bilingualism: Language and Cognition, 11, 361-363. doi:10.1017/S1366728908003581

Sera, M. D., Bales, D. W. \& Del Castillo Pintado, J. (1997). Ser helps speakers identify "real" properties. Child Development, 68, 820-831. doi:10.1111/j.1467-8624.1997.tb01964.x

Shatz, M., Diesendruck, G., Martinez-Beck, I. \& Akar, D. (2003). The influence of language and socioeconomic status on children's understanding of false belief. Developmental Psychology, 39, 717-729. doi:10.1037/0012-1649.39.4.717

Silva-Corvalán, C. (1986). Bilingualism and language change: The extensions of estar in Los Angeles Spanish. Language, 62, 587-608.

Silva-Corvalán, C. \& Montanari, S. (2008). The acquisition of ser, estar (and be) by a Spanish-English bilingual child: The early stages. Bilingualism: Language and Cognition, 11, 341-360. doi:10.1017/S136672890800357X

Slobin, D. I. (2009). Relations between paths of motion and paths of vision: A crosslinguistic and developmental exploration. En V. C. Mueller Gathercole (Ed.), Routes to language: Studies in honor of Melissa Bowerman (pp. 197-224). New York, NY: Psychology Press.

Talmy, L. (2000). Toward a cognitive semantics. Vol. 2: Typology and process in concept structuring. Cambridge, MA: Massachusetts Institute of Technology.

Taverna, A. S. \& Peralta, O. A. (2009). Desarrollo conceptual: perspectivas actuales en la adquisición temprana de conceptos. Psykhe, 18(1), 49-59. doi:10.4067/S0718-22282009000100005

Whorf, B. (1956). Language, thought and reality: Selected writings of Benjamin Lee Whorf. Editado por J. B. Carrol. Cambridge, MA: Massachusetts Institute of Technology.

Yuill, N. \& Pearson, A. (1998). The development of bases for trait attribution: Children's understanding of traits as causal mechanisms based on desire. Developmental Psychology, 34, 574-586. doi:10.1037/0012-1649.34.3.574

Fecha de recepción: Abril de 2010.

Fecha de aceptación: Septiembre de 2011. 